\title{
VI Encuentro Nacional de Medicina del Dormir
}

\author{
Luis Torre-Bouscoulet $\bowtie$
}

Academia Mexicana de Medicina del Dormir, Presidente 2013-2015.

Trabajo recibido: 25-VIII-2015; aceptado: 25-VIII-2015

Del 20 al 22 de agosto de 2015 se llevó a cabo el VI Encuentro Nacional de Medicina del Dormir; evento magno que, de forma anual, organiza la Academia Mexicana de Medicina del Dormir. «Los nuevos retos en la Medicina del Sueño" fue el tema principal que abordaron los profesores nacionales y extranjeros. Uno de los mensajes más importantes derivados del Encuentro fue que existe necesidad urgente de simplificar el proceso diagnóstico y el tratamiento de los pacientes con síndrome de apnea-hipopnea durante el sueño (SAHS).

Las nuevas evidencias disuelven los paradigmas y con ello se logra el avance científico. En este sentido, los estudios epidemiológicos recientes sobre SAHS ${ }^{1}$ han demostrado que su prevalencia es mayor a lo descrito por primera vez en 1993. En aquel trabajo, Young et al. ${ }^{1}$ informaron que el 2 y $4 \%$ de mujeres y hombres respectivamente, son portadores de SAHS. Estudios posteriores, en diversos países, demostraron prevalencias semejantes. ${ }^{2}$ Sin embargo, después de 22 años de estudios de investigación y avances biotecnológicos, contamos con herramientas diagnósticas más sensibles que, aunado a los cambios en el sistema de calificación de los eventos respiratorios, han permitido identificar apneas o hipopneas que antes podrían haber pasado inadvertidas. En un estudio reciente de base poblacional, Heinzer et al. ${ }^{3}$ demostraron una alarmante prevalencia de SAHS de $23.4 \%$ en mujeres y de $49.7 \%$ en hombres. Ese estudio se llevó a cabo en Suiza e incluyó a 3,043 individuos. Un dato importante de ese estudio es que la población incluida no era obesa (índice de masa corporal de $25.5 \mathrm{k} / \mathrm{m}^{2}$ ) y la definición de SAHS fue con un criterio estricto de 15 eventos por hora de sueño obtenido durante una polisomnografía. Lo anterior sugiere que la prevalencia de SAHS podría ser aún mayor en países con mayor frecuencia de obesidad. Estos cambios epidemiológicos así como los bien conocidos daños a la salud asociados al SAHS fueron revisados ampliamente durante el Encuentro.
Con relación al gigante problema global de salud pública que es el SAHS, los profesores señalaron que la atención médica de tantos pacientes con SAHS es, con el modelo actual, insostenible. Por ello nos mostraron evidencias acerca de la utilidad y conveniencia de los procesos simplificados para diagnosticar, tratar y dar seguimiento a los pacientes con SAHS. ${ }^{4}$ Además, compartieron la experiencia que se ha obtenido con la estrategia simplificada en condiciones de la «vida real» y nos mostraron la forma en la que la telemedicina y la creación de redes de atención médica les ha permitido hacer frente al problema creciente del SAHS. En esa red, el médico de atención primaria es el coordinador de un proceso horizontal y multidisciplinario que ofrece un servicio integral a los pacientes con SAHS. ${ }^{4}$ Las consultas médicas no presenciales han sido de gran utilidad en dicho proceso y con ella se ha hecho más eficiente la actividad asistencial de las clínicas de sueño.

Además de las conferencias, se llevó a cabo una mesa redonda acerca de la ventilación no invasiva en pacientes con insuficiencia cardíaca. Aunque es un tema controversial, la última palabra no esta dicha y debemos esperar los resultados de un gran estudio que actualmente esta en curso. Es posible que un subgrupo de pacientes con insuficiencia cardíaca se beneficie del tratamiento con ventilación no invasiva durante el sueño.

Otra actividad académica llevada a cabo durante el Encuentro fue la presentación de trabajos libres. Fueron 19 estudios; 4 de ellos se presentaron de forma oral y hubo un trabajo premiado titulado: "Comparación del cuestionario Mexicano con el cuestionario Berlín para detección del Síndrome de Apnea Obstructiva del Sueño en población mexicana» por los doctores Zaira Romero López, Liliana Camacho Ortuño, María Dolores Ochoa Vázquez, Laura Bazán Ávila, Abraham Edgar García Ramos y José Antonio Mata Marín, del Centro Médico Nacional La Raza del Instituto Mexicano del 
Seguro Social. Enhorabuena a los autores y esperamos ver pronto publicado su trabajo de investigación.

En la sesión de negocios, la Asamblea nombró como Vicepresidente a la Dra. Margarita Reyes Zúñiga y se tomó protesta a la nueva Mesa Directiva 2015-2017 que está conformada por el Dr. José Luis Carillo Alduenda, Presidente; la Dra. Martha Torres Fraga, Secretaria y la Biol. Lourdes Galicia Polo, Tesorera. A ellos nuestro reconocimiento y gratitud.

Finalmente sirvan estas líneas para manifestar nuestro agradecimiento al Instituto Nacional de Enfermedades Respiratorias Ismael Cosío Villegas por apoyar continuamente las actividades de la Academia. Muchas gracias también al Consejo Nacional de Neumología; los 10 puntos para la recertificación otorgados al Encuentro es un estímulo que favorece la educación médica continua.

A título personal, quiero invitar a toda la comunidad neumológica a que se sumen a la Academia Mexicana de Medicina del Dormir enviando su solicitud de ingreso que pueden consultar en www.medicinadeldormir. org. En poco tiempo, uno de cada dos pacientes que consulten al especialista en Medicina Respiratoria lo harán por problemas de la respiración durante el sueño. Ojalá que la Academia sea una forma de actualizar los conocimientos y competencias a favor de nuestros pacientes.

\section{REFERENCIAS}

1. Young T, Palta M, Dempsey J, Skatrud J, Weber S, Badr S. The occurrence of sleep-disordered breathing among middle-aged adults. N Engl J Med 1993;328(17):1230-1235.

2. Torre-Bouscoulet L, Vázquez-García JC, Muiño A, et al.; PLATINO Group. Prevalence of sleep-related symptoms in four Latin American cities. J Clin Sleep Med 2008;4(6):579-585.

3. Heinzer R, Vat S, Marques-Vidal P, et al. Prevalence of sleep-disordered breathing in the general population: the HypnoLaus study. Lancet Respir Med 2015;3(4):310318. doi: 10.1016/S2213-2600(15)00043-0.

4. Sánchez-de-la-Torre M, Nadal N, Cortijo A, et al.; Respiratory Medicine Research Group. Role of primary care in the follow-up of patients with obstructive sleep apnoea undergoing CPAP treatment: a randomized controlled trial. Thorax 2015;70(4):346-352. doi: 10.1136/ thoraxjnl-2014-206287.

\section{$\triangle$ Correspondencia:}

Dr. Luis Torre-Bouscoulet

Presidente de la Academia Mexicana de Medicina del Dormir 2013-2015.

Puente de Piedra Núm. 150, Torre 2 C 415 ,

Colonia Toriello Guerra, 14050,

Tlalpan, México, D.F.

El autor declara no tener conflicto de intereses. 